\title{
Fish Parasitological Studies in Bangladesh: A Review
}

\author{
K. J. ChANDRA* \\ Department of Aquaculture, Bangladesh Agricultural University, Mymensingh, Bangladesh
}

Received 12 August 2004; received in revised form 19 September 2004; accepted 12 April 2005

\begin{abstract}
Fish parasitological investigation and research performed in Bangladesh has been reviewed through study of available literature. Considerable works mainly on systematics, nature of infestation and pathology of different groups of fish parasites- protozoa, helminths and crustacea have been done. $A$ total of 290 species of parasites have so far been recorded from freshwater and marine fishes in Bangladesh. Ectoparasitic protozoans and monogenetic trematodes are recorded mainly from cultured fish species of farms. Two helminth parasites of zoonotic importance Dibothriocephalus latus and Gnathostoma spinigerum are also reported from Bangladesh fishes. Much attention has been given on caryophyllid cestodes of two catfishes Magur and Singhi. Few fish diseases of parasitic origin have been reported and studied. Commonly occurring parasitic diseases are agrulosis (fish louse), ichthyophthiriasis (white spot) and myxoboliasis. Only few attempts were taken to their control measures using simple chemicals like salt, lime, formalin, dipterex and sumithion. Recommendation has been made for the future works on parasitology for sustainable production of healthy fish.
\end{abstract}

Key words: Fish parasites, freshwater fishes, marine fishes.

\section{INTRODUCTION}

Parasite is an important group of pathogen causes infection and diseases of fish both in freshwater and marine environments. With the increasing interests in aquaculture parasitic infestations are becoming threats for fish health management and aquatic crop production throughout the world. It is therefore an essential area for proper attention to be given by the scientists for sustainable aquaculture production.

Bangladesh has a vast potential for the development of marine, estuarine and freshwater fishes. Its coastline is about $710 \mathrm{~km}$ long with about $24,800 \mathrm{sq}$. n. miles continental shelf, 2, $640 \mathrm{sq}$. n. miles territorial water and with 41,040 sq. n. miles exclusive economic zone. In addition, there are 5,332, 657 ha. of water area offered by pond, ditches, oxbow lake, reservoirs, beels, Kaptai lake and flood plain. At present there are 260 freshwater fish species, 12 species of exotic fish, 475 species of marine fish and 60 species of prawn and shrimp available in these waters. Fisheries sector contributes to GDP $5.24 \%$, animal protein supply $63 \%$ and foreign exchange earning $4.76 \%$ for the nation. If the available fisheries resources are properly exploited through development, fisheries would certainly meet the demand of animal protein for the entire nation.

\footnotetext{
*Corresponding author: Professor, Department of Aquaculture, BAU. Tel: 880-91-61920: E-mail: ffbau@mymensingh.net

(C) 2006, School of Agriculture and Rural Development, Bangladesh Open University. All rights reserved.
} 


\section{K. J. Chandra}

The various fishery development programmes depends to certain extent on the successful fish parasitological research, as the improvement of fish yield can mainly be achieved from healthy fish stock. As hosts fishes play an important role for parasites. Among the animals fishes are the most important host for maintenance of mainly helminthes. Most of the fishes have parasites. They not only serve as the host of different parasites but also serve as carrier of many larval parasitic forms that mature and cause serious diseases in many vertebrates including man. The parasites of fishes cause decrease in growth rate, weight loss and emaciation, affect yield of fish products (liver oil etc), spread human and animal diseases, postpone sexual maturity of fish and mortalities of fish.

\section{NATURE OF RESEARCH DONE IN BANGLADESH}

\section{Systematics of parasites}

Parasites of different systematic groups recorded in Bangladesh are listed in Table 1.

Protozoa: Several researchers worked out the systematics of this group of parasite. Mostly ectoparasitic protozoans- Ichthyobodo, Chilodonella, Ichthyophthirius, Trichodina reported by Hossain and Barua (1991), Hossain and Khan (1992), Chowdhury (1993) and Banu et al. (1999). Sanaullah and Ahmed (1980) reported myxobolids from Indian major carps and Chandra et al. (1996b) described myxosporeans from juvenile carps of both government and private nurseries of Mymensingh regions.

Helminths: Helminth is a big group of fish parasites belong to Trematodes (monogeneas and digeneans), cestodes, nematodes and acanthocephalans attack the fish both as external parasites (monogenean, few digeneans) and internal parasites.

Monogenea- This group of parasite mainly attacks gills and body surfaces of fishes and causes heavy damage. Considerable works have been done on systematics of monogenetic trematodes of fishes by Bashirullah (1973), Hafizuddin and Shahabuddin (1996). Recently significant works on systematics, population ecology and some aspects of histopathology have been done by Chandra et al. (2000a, 2000b), Mohanta and Chandra (2000), Mohanta et al. (2000), Hossain et al. (2000), Chandra and Jannat (2001), Ferdousi and Chandra (2002), Chandra and Yasmin (2003), Begum and Chandra (2003), Ghosh et al. (2003), and Saha et al. (2003). Most of the monogenetic trematodes are reported from freshwater fishes and only few of them are described from marine fishes. Chandra (2003) made a checklist on the monogenean fish parasites reported from Bangladesh and Indian freshwater fishes.

Digenea- This by far the most studied group among the fish parasites of Bangladesh. Bashirullah (1972) described Isoparorchis hypselobagri and noted its life cycle. A number of both marine and freshwater digenens are also reported by Bashirullah (1973), other important works are of Ahmed (1981), Bashirullah and Elahi (1972a, 1972b), Bashirullah \& Hafizuddin (1973, 1974, 1976), Chandra (1983, 1984, 1992, 1994) and Chandra and Banerjee (1992, 1993a, 1993b,). Golder and Chandra (1987), Golder et al. (1987) studied the digeneans of different fishes and Chandra (1993) recorded digenetic tremtode of estuarine fishes.

Cestodes: Fish cestodes of Bangladesh, mainly the systematics have been studied by many workers. However, histopathology, intensity of infestation and seasonal variations were also studied by several workers. Caryophyllids a special group of cestode of catfishes (magur and singhi) were given more attention for their study (Ahmed and Sanaullah, 1977, 1979; Rasheed et al., 1983, 1984, 1985; Ahmed et al., 1984; Chandra and Khatun, 1993 and Chandra et al., 1997). Khusi et al. (1993), and D'Silva and Khatoon (1997) identified few marine cestodes. Chandra (1992) described trypanorhynchid larval cestodes from estuarine fishes of eastern part of Bay of Bengal, Bangladesh. Uddin et al. (1980) described Dibothriocephalus latus from Bambay duck (B. loitta), a marine fish of Bay of Bengal and Chowdhury et al. (1982) reported Diphyllobothrid plerocercoid from meni fish of Mymensingh. However, some authors (Hoffman, 1968, Moravec, 1998) termed its presence in Bangladesh as improbable.

Acanthocephala: This is a small group of fish parasite though causes serious injuries and secrets toxins to infested fish. It has received very little attention by Bangladeshi scientists. Ahmed and 
Rouf (1981), Ahmed and Begum (1978), Chowdhury et al. (1982), Chandra (1985, 1987, 1992a, 1993), Chandra and Rahman (1988) and few others contributed on the systematics of this group fish parasite and described several species.

Nematode: A good number of nematode species have been described equally from marine and freshwater fishes. Bashirullah (1973) reported several nematode species from marine fish. Chandra (1992b) listed the nematodes recorded from freshwater fishes of Indian sub-continent. Bashirullah (1972, 1973, 1974a, 1974b), Ahmed and Begum (1978) and Ahmed and Rahman (1977) studied the systematics of several nematode worms. Bashirullah and Ahmed (1976a, 1976b) observed development of Camallanus adamsi and Spirocamallanus intestinecolisi in the copepod intermediate host. Chandra and Modak (1995) observed the development, activity and penetration efficiency of first stage larvae of Procamallanus heteropneustus in copepods. Mandal (1995) described few nematodes from lizardfishes of Bay of Bengal. Bashirullah (1973) and several other workers (Khanum et al., 1996, AKhter et al., 1997) reported Gnathostoma spinigerum from a dozen of fish species. This nematode is the cause of gnathostomiasis, a serious disease of man.

Crustacea: These include parasitic copepods, isopods, argulids etc. parasitising the gills and skin of fishes. They often pose great problem in fish nurseries and culture systems. No systematic works were conducted on crustacean parasites of fishes of Bangladesh. But there are several reports particularly of Argulus (fish louse) infesting nurseries and other cultural fishes. Golder et al. (1983) reported them from a fish farm survey and Rahman (1968) described from tilapia, chital, khalisha and singhi. Chandra et al. (2004) reported fish louse in the cultured major carps of Mymensingh. Lernaea (anchor worm) was recorded by Hossain et al. (1978) and isopods by Ahmed and Rahman (1976).

Table 1. List of fish parasites with their hosts recorded in Bangladesh

\begin{tabular}{|c|c|}
\hline Name of parasite & Name of host \\
\hline $\begin{array}{l}\text { Protozoa } \\
\text { Ichthyobodosp. }\end{array}$ & Rui, Catla \\
\hline Chilodonellasp. & Catla, Tila \\
\hline Ichthyophthirius multifiliis & Catla, Rui, Mrigal \\
\hline Ichthyophthirius sp. & Mrigal \\
\hline Apisoma sp. & Carps \\
\hline Trichodinasp. & 10 spp of carps \\
\hline Tripartiella sp. & $\begin{array}{l}\text { Lata, Mrigal, } \\
\text { Magur, Rui, } \\
\text { Baim, Carp }\end{array}$ \\
\hline Myxobolussp. & $\begin{array}{l}\text { Sarputi, Major } \\
\text { carps, Magur, } \\
\text { Silver carp }\end{array}$ \\
\hline Thelahanella dogieli & Rui \\
\hline \multicolumn{2}{|l|}{ Monogenea } \\
\hline Dactylogyrus labei & Major carps \\
\hline D. kalyanensis & Catla \\
\hline D. mrigali & Mrigal \\
\hline D. caltius & Catla \\
\hline D. chauhanus & Mrigal \\
\hline D. yogendrai & Mrigal \\
\hline D. angularis & Puti \\
\hline D. subtilis & Puti \\
\hline D. brevitignus & Puti \\
\hline D. lampam & Sarputi \\
\hline D. lamellatus & Grass carp \\
\hline D. hypophthalmichthys & $\begin{array}{l}\text { Silver carp, Miror } \\
\text { carp }\end{array}$ \\
\hline D. siamensis & Thaisarputi \\
\hline
\end{tabular}

\begin{tabular}{ll}
\hline Name of parasite & Name of host \\
\hline \hline D. mymensinghi & Puti \\
D. bangladeshi & Puti \\
D. ogawai & Puti \\
D. tripathii & Puti \\
D. gussevi & Puti \\
D. glossogobii & Bele \\
D. cirrhini & Bele \\
D. multispiralis & Puti \\
D. mrigali & Mrigal \\
D. vastator & Magur \\
D. hypothalamus & Silver carp \\
D. minutus & Carpio \\
D. rebai & Reba \\
Neodactylogyrus chandrai & Bele \\
Glossodactylogyrus bangladeshi & Bele \\
Silurodiscoides octotylus & Boal, Garua \\
S. indicus & Boal \\
S. sudhakari & Vacha \\
S. siamensis & Pangus \\
S. parvulus & Tengra, Batasi \\
S. parvulus & Tengra, Batasi \\
Silurodiscoidessp. & Vacha \\
Silurodactylogyrus chandai & Chanda \\
Mizelleus wallogonius & Boal \\
M. indicus & Boal \\
Heteronchocleidus buschkieli & Khalisha \\
H. colisai & Khalisha \\
H. bangladeshi & Khalisha \\
H. anabasi & Koi \\
&
\end{tabular}




\begin{tabular}{|c|c|}
\hline Name of parasite & Name of host \\
\hline "Ancylodiscoides indicus & Boal \\
\hline A. notopterus & Chital \\
\hline Sprostonia wallagonia & Boal \\
\hline S. asiatica & Kajali \\
\hline Neosprostoniasp. & Vacha, Garua \\
\hline Thaparocleidus kao & Boal \\
\hline $\begin{array}{l}\text { Hamatopenduncularia } \\
\text { lucknowensis }\end{array}$ & Boal \\
\hline Bychowskyella tchangi & Magur \\
\hline Bychowskyellasp. I & Vacha \\
\hline Bychowskyellasp. II & Vacha, Garua \\
\hline Quadricanthus kobiensis & Magur \\
\hline Cichlidogyrus bangladeshi & Tilapia \\
\hline C. chandrai & Tilapia \\
\hline Actinocleidus mulleri & Tilapia \\
\hline Oreochromogyrus mymensighi & Tilapia \\
\hline Bifurcohaptor indicus & Tengra, Pangus \\
\hline Haplocleidus xenentodoni & Kakila \\
\hline Urocleidus raipuirensis & Guchi \\
\hline Cornudiscoides proximus & Tengra \\
\hline C. vittati & Tengra \\
\hline Ancyrocephalus daniconis & Chela \\
\hline A. ambassi & Chanda \\
\hline A. chakrabortii & Darkina \\
\hline Pseudoanthocotyle parvovskyi & Champa \\
\hline Pricea multae & Maitta \\
\hline Megamicrocotyle pagelli & Ilish \\
\hline Choricotyle sp. & Ilish \\
\hline Gyrodactylus sp. & $\begin{array}{l}\text { Puti, Rui, Carpio, } \\
\text { Thaisarputi }\end{array}$ \\
\hline \multicolumn{2}{|l|}{ Digenea } \\
\hline Clinostomum complanatum & Magur, Singhi \\
\hline C. giganticum & Meni \\
\hline Clinostomum sp. & Magur, Singhi \\
\hline Euclinostomum heterostomum & Lata, Shol \\
\hline E. multicaecum & Lata, Shol, Singhi \\
\hline Euclinostomumsp. & $\begin{array}{l}\text { Pabda, Singhi, } \\
\text { Meni }\end{array}$ \\
\hline Neascus sp. & Major carps \\
\hline Posthodiplostomum minimum & Magur \\
\hline Bucephalus mystusi & Air \\
\hline Bucephalussp. & Silong, Air \\
\hline Bucephalopsis thapari & Pabda \\
\hline Neobucephalopsis bagarius & $\begin{array}{l}\text { Garua, Vacha, } \\
\text { Pabda }\end{array}$ \\
\hline Prosorhynchoides spinosiensis & Garua \\
\hline Prosorhynchoides aspinosiensis & Garua \\
\hline Polyorchitrema inglishi & Garua \\
\hline P. clupisomus & Vacha \\
\hline Prosorhynchoides sp. & Vacha, Air \\
\hline Faustula brevichrus & Ilish \\
\hline Faustulasp. & Ilish \\
\hline Steringotremasp. & Puti \\
\hline Rhynchopharynx paradoxa & Guchi \\
\hline Aphanurus stossichi & Ilish, Nailya \\
\hline
\end{tabular}

\begin{tabular}{|c|c|}
\hline Name of parasite & Name of host \\
\hline Opisthadena sp. & "Nailya \\
\hline Gonocera crassa & Pabda \\
\hline Genarchopsis bengalensis & Lata \\
\hline Genarchopsis bashiri & Singhi \\
\hline G. dasus & Singhi, Bele \\
\hline G. lobota & Raga \\
\hline G. microcotyle & Lata \\
\hline G. ozaki & Lata \\
\hline G. wallagoni & Boal \\
\hline Genarchopsis sp. & Silong, Garua, Bele \\
\hline Philopinnasp. & Lata, Singhi \\
\hline Lecithocladium excisum & Rup chanda \\
\hline L. harpodontis & Loitta \\
\hline L. magnacetabulum & llish \\
\hline L. megalaspis & Kauwa \\
\hline L. seriolellae & Kauwa \\
\hline Lecithocladium sp. & Rup chanda \\
\hline Himiurus appendiculatus & Nailya \\
\hline Himiurus sp. & Nailya \\
\hline Isoparorchis hypselobagri & Catfish 16 FW fish \\
\hline Haploporus sp. & Mugil \\
\hline Psilostomum sp. & Bhetki \\
\hline Alocreadium banglensis & Baim \\
\hline A. glossogobium & Bele \\
\hline A. handiai & $\begin{array}{l}\text { Lata, Garua, } \\
\text { Bele, Singhi }\end{array}$ \\
\hline A. madheri & Pabda \\
\hline A. mehrai & Baim, Guchi \\
\hline A. minimum & Koi \\
\hline A. mymensinghi & Singhi \\
\hline A. ovatum & Bele \\
\hline Allocreadium sp. & Pangus \\
\hline Macroleithus sp. & Air \\
\hline Coitocaecum sp. & Air \\
\hline Crowcrocaecum channai & Gazar \\
\hline Neopecoelina sharanpuriensis & Koi, Singhi, Lata \\
\hline Neopecoelina sp. & Singhi, Lata \\
\hline Opegaster beliyai & Bele, Singhi \\
\hline Opegaster sp. & Bele \\
\hline Catylogonoporus orfeum & Baim, Guchi \\
\hline Eucreadium daccai & Lata \\
\hline Macvicaria crassigula & Singhi \\
\hline Podocotyle atomon & Meni \\
\hline Opistholebes sp. & Punti \\
\hline Acanthocolpus liodorus & Chhuri \\
\hline A. luehei & Chhuri \\
\hline Mogomtistrema attu & Lata, Guchi \\
\hline Opithorchis bagarius & Baghair \\
\hline Opisthorchis sp. & Rita \\
\hline Phyllodistomum chauhani & Vacha \\
\hline$P$. folium & Magur,Bele,Lata \\
\hline$P$. yousufzaii & Rita \\
\hline Phyllodistomum sp. & Lata \\
\hline Pleurogenes attui & Boal \\
\hline P. pabdai & Pabda \\
\hline
\end{tabular}




\begin{tabular}{|c|c|}
\hline Name of parasite & Name of host \\
\hline P. notopteri & Chital \\
\hline Eumanesia sp. & Singhi \\
\hline Masenja dayali & Magur \\
\hline Orientocreadium batrachoides & Magur, Singhi \\
\hline Asymphylodora indica & Lata \\
\hline Masenia sp. & Tengra \\
\hline Palaeorchissp. & $\begin{array}{l}\text { Lata, Vacha, } \\
\text { Garua, Singhi }\end{array}$ \\
\hline \multicolumn{2}{|l|}{ Cestoda } \\
\hline Bovienia serialis & Magur \\
\hline Bovieniasp. & Magur \\
\hline Djombangia penetrans & Magur \\
\hline Lytocestus birmanicus & Magur \\
\hline L. indicus & Magur \\
\hline L. latevitellarium & Magur \\
\hline L. parvulus & Magur \\
\hline Lytocestussp. & Magur \\
\hline Monothrioides sp. & Magur \\
\hline Bialovarium sp. & Singhi \\
\hline Caryophyllaeus sp. & $\begin{array}{l}\text { Vacha, Garua, } \\
\text { Silong, Air }\end{array}$ \\
\hline Lytocestoides sp. & Pabda, Garua \\
\hline Capingentoides batrachii & Magur \\
\hline $\begin{array}{l}\text { Pseudocaryophyllaeus } \\
\text { heteropneutes }\end{array}$ & Singhi \\
\hline P. indica & Magur \\
\hline Pseudocaryophyllaeus sp. & Magur \\
\hline Pseudolytocestus clariae & Magur \\
\hline Nybelinia sp. & Chhuri \\
\hline Poecilancistrium ilisha & \|lish \\
\hline Pterobothrium acanthotruncalum & Guji \\
\hline Gangesiasp. & Garua \\
\hline P. heterocanthum & Ilish \\
\hline P. lintoni & Bhetki \\
\hline Dasyrhynchus indicus & Bhetki \\
\hline Gynorhynchus gigas & Bhetki \\
\hline Gymnorhynchus sp. & Pangus, Silong, Ilish \\
\hline Calliotetrarhynchus gracilis & Bhetki \\
\hline Diculiceps pileatum & Telchitta \\
\hline $\begin{array}{l}\text { Bothriocephalus cuspidatus } \\
\text { Bothriocephalus sp. }\end{array}$ & $\begin{array}{l}\text { Lata, Shol, } \\
\text { Meni }\end{array}$ \\
\hline Polyonchobothrium sp. & Lata, Shol \\
\hline Senga ophiocephalina & Meni \\
\hline Taphrobothrium japonese & Lata, Shol \\
\hline Anchistrocehalus sp. & Lata, Shol \\
\hline Marsipometra parva & Baim \\
\hline Ligula intestinalis & Singhi \\
\hline \multicolumn{2}{|l|}{ Nematoda } \\
\hline Eustrongylides tubifex & Pabda \\
\hline Eustrongylidessp. & Pabda, Garua \\
\hline Capillaria sp. & !0 spp. of FW fish \\
\hline Cosmoxynemoides sp. & Khosha \\
\hline Ascaridiasp. & $\begin{array}{l}\text { Lata, Magur, } \\
\text { Singhi, Meni }\end{array}$ \\
\hline & \\
\hline
\end{tabular}

\begin{tabular}{|c|c|}
\hline Name of parasite & Name of host \\
\hline $\begin{array}{l}\text { Goezia sp. } \\
\text { Contracaecum aori } \\
\text { C. brevicaecum } \\
\text { Contracaecum sp. }\end{array}$ & $\begin{array}{l}\text { Boal, Lombu } \\
\text { Air } \\
\text { Sawfish } \\
19 \text { spp. of FW fish }\end{array}$ \\
\hline $\begin{array}{l}\text { Heterohylum sp. } \\
\text { Paranisakis sp. }\end{array}$ & $\begin{array}{l}\text { Flatfish, llish } \\
\text { Nailya }\end{array}$ \\
\hline Rapidaskaris panijii & Tular dandi \\
\hline $\begin{array}{l}\text { Rapidskaris sp. } \\
\text { Terranova sp. }\end{array}$ & $\begin{array}{l}\text { Sardine, Tapasi } \\
\text { Eusphyra }\end{array}$ \\
\hline Pseudoanisakissp. & Eusphyra \\
\hline Ascaris sp. & Eusphyra \\
\hline Dujardinaskaris sp. & Kukurjib \\
\hline Porrocaecum trichiuri & Serbuti \\
\hline Porrocaecum sp. & Meni, Vacha \\
\hline Falcaustra brevicaudatum & Pabda \\
\hline Faulcaustrasp. & Kajuli \\
\hline Cucullanus pangasius & Pangus \\
\hline Cucullanus sp. & Rita, Air \\
\hline Dichelynesp. & Rita \\
\hline Paragendria bagarii & Kakila \\
\hline P. wallagonia & Koi \\
\hline Paragendriasp. & Air \\
\hline Buckleynemasp. & Air \\
\hline Paraquimperia sp. & Kukurjib \\
\hline Pingus aori & Air \\
\hline Quimperia sp. & Sardine, Upenes \\
\hline Camallanus anabantis & Koi \\
\hline C. magna & Guc \\
\hline C. pearsi & Koi,Lata \\
\hline Camallanus trichiuris & Chhuri \\
\hline C. truncatus & Tengra \\
\hline C. xenentodoni & Baim, Kakila \\
\hline Camallanus sp. & $\begin{array}{l}\text { Puti, Lata, } \\
\text { Magur, Bele }\end{array}$ \\
\hline Neocamallanus ophiocephali & Lata, Shol \\
\hline N. vachi & Vacha \\
\hline Neocamallanus sp. & Lata, Shol, Gajar \\
\hline Paracamallanus sweeti & Lata, Shol \\
\hline Procamallanus alii & Pabda \\
\hline P. berdii & Dat \\
\hline P. cancillus & Kakila \\
\hline P. clarius & $\begin{array}{l}\text { Magur, Singhi, } \\
\text { Baim, Pabda }\end{array}$ \\
\hline P. mysti & 9spp. of FW Catfish \\
\hline P. spiculogubernaculus & $\begin{array}{l}\text { Lata, Singhi, } \\
\text { Boal, Tengra }\end{array}$ \\
\hline Procamallanus sp. & Lata, Singhi, Boal \\
\hline Echinocephalus sp. & Lata, Singhi, Magur \\
\hline Gnathostoma spinigerum & 12 spp. of FW fish \\
\hline Heliconema brevispiculum & Gajar \\
\hline Proleptus inflatus & Baim \\
\hline Pseudoproleptus vestibulus & Baim \\
\hline Spinitectus indicus & Garua, Vacha, Boal \\
\hline Rhabdochona bagarii & Baghair \\
\hline R. magna & Rita \\
\hline
\end{tabular}




\begin{tabular}{ll}
\hline Name of parasite & Name of host \\
\hline \hline Acanthocephala & \\
Heterocentis plotosi & Gangmagur \\
Echninorhynchus kushiroensis & Bele \\
Sachalinorhynchus sp. & Rui \\
Hypoechinorhynchus sp. & Golsha \\
Cleavius secundus & Air \\
Serrasentis sagittifer & Bhetki \\
Acanthogyrus acanthogyrus & Catla \\
A. dattai & Puti \\
A. indicus & Phasa \\
A. tilapiae & Lata, Magur \\
Acanthogyrus sp. & Vacha, Magur \\
Pallisentis allahabadi & Lata \\
P. goboes & Lata, Magur, \\
& Garua, Pabda \\
P. garuai & Garua, Silong \\
P. nagpurensis & Lata, Shol \\
P. nandai & Bele, Meni \\
& Lata, Shol, Meni, \\
& Vacha
\end{tabular}

\begin{tabular}{ll}
\hline Name of parasite & Name of host \\
\hline \hline Pallisentis sp. & 13 spp. of FW fish \\
Neoechinorhynchus aminuhaquel & Tengra \\
N. topseyi & Topsey \\
Neoechinorhynchus sp. & $\begin{array}{l}\text { Tengra, Meni, } \\
\text { Kukurjib }\end{array}$ \\
Annelids & \\
Piscicola sp. & Magur \\
Crustacea & \\
Argulus bengalensis & Vacha \\
A. foliaceus & Rui \\
Argulus sp. & 11 spp. of FW fish \\
Lernaea cyprinacea & Lata, Shol, Puti, \\
& Khalisha \\
Lernaea sp. & Carpio \\
Ergasilus sp. & Kukurjib \\
Lernaeocera sp. & Singhi \\
Ichthyoxenus amurensis & Batasi \\
Cymothoidae gen sp. & Kukurjib \\
Isopoda gen. sp. & Catla, Silong \\
&
\end{tabular}

\section{SURVEY OF PARASITIC DISEASES}

Ali (1968) made a brief survey on the diseases and parasites from different regions of Bangladesh (then East Pakistan) and recorded argulosis in farm fishes. Rahman $(1967,1968)$ described mortalities of carp due to argulosis. Rahman and Ali (1968) further noted the prevalence of nematode (Procamallanus) and acanthocephala (Pallisentis) from different waterbodies of Bangladesh (then East Pakistan). Ahmed (1982) observed skin myxoboliasis in a major carp (Labeo rohita). Ahmed and Rahman (1977) studied the pathogenecity of some nematodes in flat fishes of Bay of Bengal. Ahmed and Sanaullah (1979) observed intestinal lesions induced by caryophyllid cestodes in magur. Banu et al. (1999) surveyed the parasitic diseases of freshwater fish in nursery operations and decribed few ectoparasitic diseases. Chandra et al. (1996) made a clinico-anatomical study on yellow grub disease of singhi. Mohanta (1995), Hossain (1995) studied the histopathogy of dactylogyrosis. Sanaullah (1984) reported black spot and leech infestation in freshwater fish. Sanaullah and Ahmed (1980) reported myxoboliasis in carps and Chandra et al. (2000) investigated myxoporean disease of jevenile carps of nurseries of Mymensingh. Muniruzzaman (2002) studied the ichthyophthiriasis from carps and Chandra et al. (2004) also recorded argulosis in fish ponds of Bangladesh Agricultural University campus.

\section{PARASITIC DISEASES AND CONTROL MEASURES}

The works on erradication and curative measures of parasitic diseases of fishes have been very few and far between. For external, infection particularly for argulosis few published data and several reports of popular articles are available on their control measures. Rahman (1969) mentioned that lime and potassium permanganate would be useful for argulosis. Chandra et al. (2004) used sumithion for controlling argulosis in BAU fish farms. Common salt, dipterex and lime were used in many cases. Sanaullah (1993) suggested to control leech and myxobolus infection with lime and salt. Muniruzzaman et al. (2002) has successfully controlled ichthyophthiriaisis (white spot) with salt and potassium permanganate in several fish farms. However, fish farmers treat some diseases without systematic approach. Many organizations follow their own technique.

The damage caused by monogenetic trematodes producing dactylogyrosis and gyrodactylosis is not yet known in Bangladesh waters. No treatments have yet been taken in Bangladesh for the diseases caused by cestodes, acanthocephalans, nematodes and digeneans or other endoparasites. 
In some cases prophylactic measures like good pond management i.e., avoiding of overstocking, pond drying and limings were suggested to control parasitic diseases.

For chemotherapy, chemicals so far known to be used are : Salt, Lime, Salt + lime, Potassium permangante, Copper sulphate, Malachite green, Dipterex, Dipterex + lime, Formalin, Sumithion.

\section{CONCLUSION AND RECOMMENDATION}

From the foregoing account it would be clear that information on fish parasites is very meagre and no planned investigations have been carried out so far in Bangladesh. Knowledge about loss incurred in the fish production and fish products due to parasites and parasitic diseases is not available. Parasites that infest fishes occur in a wide variety of forms and may have complicated life histories. Insufficient information of many of the most common parasites greatly handicaps the efforts at their possible conrol. It may be emphasized that ichthyo-parasitological investigations must be carried out in all river systems, large lakes, haors, reservoirs and maritime belts. It would be better to compile the parasite concentration index and parasite frequency index for different water bodies that will give a clear picture of nature of parasitism. However, some of situations should be tackled on priority basis for healthy growth and better production of fish.

1. Assessment of parasitism and their effect on fishes

2. Ecology and geographical distribution of important parasites and their hosts

3. Studies on the life-history and physiology of parasites

4. Chemical and biological control

From aquaculure point of view the specific recommedations are as follows:

1. Baseline studies on identification of parasites of hatchery, nursery, rearing and culturing ponds

2. Influence of environmental factors (physico-chemical and biological) and agricultural pesticides which increase the susceptibility of fish towards parasitic infestation

3. Histopathological studies of fish diseases casused by parasites which could be used as key indicator of particular disease

4. Preventive and therapeutic measures suitable and appropriate for farms particularly using locally available materials

\section{LITERATURE CITED}

Ahmed, A. T. A. 1981. Helminth infection in freshwater fishes of Bangladesh. Fish Pathol 15, 229-236.

Ahmed, A. T. A. 1982. Skin myxoboliasis of a major carp (Labeo rohita) in Bangladesh. Fish Health News 2, 1-2.

Ahmed, A. T. A. and Begum, R. 1978. Studies on the distribution of some endoparasitic helminths in six freshwater fishes of Dacca and Barisal. Bangladesh J Aquacult 1, 52-60.

Ahmed, A. T. A. and Rahman, M. S. 1977. Distribution of some nematode and crustacean parastites in Psettodes erumei (Bloch) and Cynoglossus macrolepidotus (Bleeker) in the Bay of Bengal. $J$ Asiatic Soc Bangladesh (Sci) 2, 7-14.

Ahmed, A. T. A. and Rouf, A. J. M. A. 1982. Acanthocephalan parasites of freshwater and estuarine fishes of Bangladesh. Proc $3^{\text {rd }}$ Nat Zool Conf 1981 Dacca. pp. 118-125.

Ahmed, A. T. A. and Sanaullah, M. 1977. Studies on the distribution of some metazoan parasites of Heteroneustes fossilis (Bloch) and Clarias batrachus (L.) in Bangladesh. Bangladesh J Zool 5, 117--123.

Ahmed, A. T. A. and Sanaullah, M. 1979. Pathological observations of the intestinal lesions induced by caryopyllid cestodes in Clarias batrachus (Linneaus)(Siluriforemes:Clariidae). Fish Pathol 14, 1-7.

Ahmed, A. T. A., Roy, P. and Mustafa, G. 1984. Organal distribution of some cestode parasites and their percentage of infection in two catfishes. J Asiatic Soc Bang (Sci) 10, 1-6.

Ahmed, G. and Banu, A. N. H. 2001. Investigation on diseases of some smalll indigenous freshwater fishes of Bangladesh. Final Rep. BARC, Dhaka pp.67.

Akhtar, M., D'Silva, J. and Khatun, A. 1997. Helminth parasites of Anabas testudeneus Bloch in Bangladesh. Bangladesh J Zool25, 135-137.

Ali, M. Y. 1968. Investigation on fish diseases and parasites in East Pakistan. Bull Office Intern Epizoot 69, 1517-1521. 


\section{K. J. Chandra}

Banerjii, M. and Chandra, K. J. 1992. Digenetic trematodes from freshwater fishes of Mymensingh, Bangladesh. Family Allocreadiidae. Riv Di Parassitol 53, 361-371.

Banu, A. N. H., Khan, M. H., Hossain, M. A. and Azim, M. E. 1999. Parasitic diseases of freshwater fish in nursery operation system of Bangladesh, Abstract. No 61. In Book of Abstract. Fourth Symposium on Diseases in Asian Aquaculture,"Aquatic Animal Helath for Sustainabulity," 22-26 November, 1999. Cebu International Convocation Centre, Cebu City, Philippines. Fish Helath Section, Asian Fisheries Society.

Bashirullah, A. K. M. 1972. On the occurrence of the trematode, Isoparorchis hypselobagri (Billet, 1898) in fishes and notes on its life history. Norwegian J Zool 20, 209-212.

Bashirullah, A. K. M. 1973. A brief survey of the helminth fauna of certain marine and freshwater fishes of Bangladesh. Bangladesh J Zool1, 63-81.

Bashirullah, A. K. M. 1974a. Notes on Spirocamallanus olseni Bashirullah, 1973. Am Nat 92,256.

Bashirullah, A. K. M. 1974b. Two new nematode species of Camallanus Ralliet and Hendry, 1915 from freshwater fishes of Dacca, Bangladesh. Norwegian J Zool 22, 57-60.

Bashirullah, A. K. M. and Ahmed, B. 1976a. Development of Camallanus adamsi Bashirullah, 1974 (Nematoda: Camallanidae)in cyclopoid copepods. Can JZool 54, 2055-2060.

Bashirullah, A. K. M. and Ahmed, B. 1976b. Larval development of Spirocamllanus intestinecolas (Bashirullah, 1973) Bashirullah, 1974 in copepods. Riv Di Parassitol 37, 303-311.

Bashirullah, A. K. M. and Elahi, K. M. 1972a. On two new two species of GenarchopsisOzaki, 1925 from freshwater fishes of Daca, Bangladesh. Riv Di Parassit 33, 277-280.

Bashirullah, A. K. M. and Elahi, K. M. 1972b. Three trematodes (Allocreadiidae) from the freshwater fishes of Dacca, Bangladesh. Norwegian JZool 20, 205-208.

Bashirullah, A. K. M. and Hafizuddin, A. K. M. 1973. Two new nematodes (Camallanidae) from freshwater fishes of Bangladesh. Riv Di Parassit 34, 115-119.

Bashirullah, A. K. M. and Hafizuddin, A. M. M. 1976. Digenetic trematodes from freshwater fishes of Bangladesh. Riv Di Parassit 37, 35-39.

Bashiruulah, A. K. M. and Hafizuddin, A. K. M. 1974. Two new nematode species of Procamallanus Baylis, 1923 from fishes of Dacca, Bangladesh. Norwegian J Zool 22, 53-55.

Begum, M. M. and Chandra, K. J. 2003. Investigation on monogenetic trematodes of Mystus vittatus, Ailia coila and Esomus danricus of Mymensingh. J. Bangladesh Agril.Univ. 1(1), 87-98.

Chandra, K. J. 1983. A note on the metacercaria of Euclinostomun multicaecum Tubangui \& Musilungan, 1985 from freshwater fishes of Bangladesh, Bangladesh J Aquacult 25, 91- 93.

Chandra, K. J. 1984. Nature of Euclinostomum multicaecum Tubangui \& Musilungan, 1935 (Clinostomidae: Digenea) infestation in Channa punctata (Bloch). Bangladesh Vet $J$ 18, 49-54.

Chandra, K. J. 1985. Incidence and intensity of infestation of Pallisentis ophiocephali (Thapar) on the host Channa punctatus(Bloch). J Asiatic Soc Bangladesh 11, 47-54.

Chandra, K. J. 1992a. Studies on the helminth parasites, infections and diseaes of some freshwater and estuarine fishes of Bangladesh. BAU Res Prog 6, 402-408.

Chandra, K. J. 1992b. Records of nematode parasites of freshwater fishes of Indian subcontinent. Proc First Ann Conf, Bangladesh Soc Parasitolpp. 52-71.

Chandra, K. J. 1993. Helminth parasites of certain freshwater and marine fishes of Bangladesh. BAU Res Prog 7, 543-554.

Chandra, K. J. 1994. Infections, concurrent infections and fecundity of Procamallanus heteropneustus Ali, parasitic to the fish Heteropneustes fossilis. Environ Ecol 12, 679-684.

Chandra, K. J. 1998. Seasonal biology of Procamallanus heteropneustus Ali, 1957 in fish Heteropneustes fossilis of Mymensingh. Bangladesh J Aquacult 20, 7-14.

Chandra, K. J. and Baherjii, M. 1992. Opisthorchis bagarius n. sp. (Digenea : Trematoda) from a freshwater fish Bagarius bagarius of Mymensingh. Riv Di Parassitol 53, 373-377.

Chandra, K. J. and Banerjii, M. 1993. Digenetic trematodes from freshwater fishes of Mymensingh. Families Clinostomidae and Hemiuridae. Riv Di Parasitol 54, 81-91.

Chandra, K. J. and Banerjii, M. 1993. Three digenetic trematode parasites from freshwater fishes of Mymensingh. Riv Di Parassitol 54, 71-79.

Chandra, K. J. and Golder, M. I. 1987. Effect of helminth parasites on a freshwater fish Nandus nandus. Environ Ecol 5, 333-336. 
Chandra, K. J. and Khatun, M. R. 1993. A new species of caryophyllid cestode from Heteropneustes fossilis of Mymensingh. Riv Di Parassitol 54, 235-239.

Chandra, K. J. and M. S. Jannat, M. S. 2002. Monogenean gill parasites of manor carps from different fish farms of Mymensingh. Bangladesh J Fish Res6, 43-52.

Chandra, K. J. and Modak, P. C. 1995. Activity, ageing and Penetration of the first stage larvae of Procamallanus heteropneustus Ali, 1957 (Nematoda: Camallanidac). Asian Fish Sci 8, 95-101.

Chandra, K. J. and Rahman, M. A. 1988. A new host record for Pallisentis ophiocephali (Thapar, 1930). Indian J Parasitol 12, 37-38.

Chandra, K. J. and Yasmin, R. 2003. Some rare and new monogenetic trematodes from air-breathing freshwater fishes of Bangladesh. Indian J Anim Sci 73, 113-118.

Chandra, K. J., Alam, M. N. and Baki, M. A. 1996a. Clinico-anatomical studies on yellow grub disease of singhi, Heteropreustes fossilis (Bloch) of Mymensingh. Bangladesh. J Agric 21, 87-94.

Chandra, K. J., Begum, A. A., Ahmed, G. U. and Wootten, R. 1996b. Infection of Myxosporean ectoparasites of juvenile carps in nurseries of Mymensingh Bangladesh. Bangladesh J Aquacult 18, 39-44.

Chandra, K. J., Das, A. K., Alim, M. A. and Barai, A. K. 2004. Lice (Argulus foliaceus) on carp of Bangladesh Agricultural University experimental pond. Bangladesh Veterinan 21, in press.

Chandra, K. J., Islam, M. Z. and Wootten, R. 1997. Some aspects of association and development of Lytocestus indicus Moghe in Catfish Clarias batrachus (Lin.). Bangladesh J Fish Res 1, 31-38.

Chandra, K. J., Mohanta, S. K., Hossain, M. M, Nahar, S., Yasmin, R. and Paul, S. K. 2000a. A study on the prevalence of monogenetic ectoparasites of freshwater fishes. BAU Res. Prog 11, 134-143.

Chandra, K. J., Paul, R. K. and Islam, M. A. 2000b. Monogenean ectoparasites of Wallago attu in freshwater fishes of Mymensingh, Bangladesh Bangladesh J Agric 25, 2000 (in press).

Chowdhury, M. B. R. 1993. Research priorities for microbial fish and its control in Bangladesh. In "Research Priorities in Bangladesh for Fish Health, Disease Prevention and Pathology" (Tollervey, Ed.), A one-day ODA/BAU workshop held at the Faculty of Fisheries, BAU, Mymensingh, 17 May, 1993. pp. 8-11.

Chowdhury, M. B. R., Haque, A. K. M. and Islam, M. A. 1982. Incidence of diphyllobothriid larva and Pallisentis nandai in Nandus nandus (Hamilton-Buchanan) fish. Bangladesh J Agricult Sci 9, 191-197.

D'Silva, J. and Khatun, S. M 1997. Helminth parasites of two clupeid fishes from the Bay of Bengal, Bangladesh. J NOAMI14, 27-37.

Ferdousi, U. K. and Chandra, K. J. 2002. Monogenean gill parasites of Oreochromis niloticus (Linnaeus) and Oreochromis mossambicus (Peters) (Osteichthyes, Cichlidae) from Mymensingh, Bangladesh. Riv Di Parassit 64, 49-60.

Ghosh, P. K., Chandra, K. J. and Saha, P. K. 2003. Monogenean intestation in indigenous small fishes of Bangladesh. Riv Di Parassit 64, 189-201.

Golder, M. I. and Chandra, K. J. 1987. Infestation of Isoparorchis hypselobagri on the host fish Nandus nandus. Environ Ecol5, 337-341.

Golder, M. I. and Chandra, K. J. and Rahman, A. K. A. 1987. Helminth parasitism in Nandus nandus (Hamilton). Bangladesh J Fish 10, 11-22.

Hafizuddin, A. K. M. and Shahabuddin, M. 1996. Parasitic monogeneans from freshwater fishes of Commilla, Bangladesh. Chittagong Univ Stud Sci20, 113-126.

Hoffman, G. L. 1968. "Parasites of north American freshwater fishes". Comstock Publishing Associates, Itthaca and London, $539 \mathrm{p}$.

Hossain, M. A. and Barua, G. 1991. Diseases of cultured fish and their control. In "Improved Fish Culture Management Practices" (M. V. Gupta Ed.), Trainer's Training Manual for Fisheries Extension Officers. Fisheries Research Institute, Mymensingh. pp. 175-191.

Hossain, M. A. and Khan, M. H. 1992. Prevalence of ectoparasites of carps in Bangladesh nurseries. In Third Asian Fisheries Forum, October 26-30, 1992, Singapore Abstracts. Asian Fisheries Society. pp.51.

Hossain, M. D., Islam, M. A. and Huq, M. M. 1978. Investigation into the parasitism of Bangladesh Agricultural University pond fishes. Bangladesh J Aquacult 1, 15-20.

Hossain, M. M. 1995. Studies on the monogenean gill parasites of three Puntius species of Mymensingh. M.S. thesis in Aquaculture, BAU, Mymensingh. pp. 66-70.

Hossain, M. M., Chandra, K. J. and Mohanta, S. K. 2000. Monogenetic trematodes from Puntius stigma (Valenciennes) of Mymensingh, Bangladesh. Riv Di Parassit. 61, 217-224. 


\section{K. J. Chandra}

Khanum, H., Ahmed, A. T. A. and Zaman, Z. 1996. Endoparasite community of two species go genus Ompak Lacepede. J Bengal Nat His Soc NS 15, 32-36.

Khusi, K., Khatun, A. and D'Silva, J. 1993. Cestode parasites from elamobrach fishes in the Bay of Bengal . p.12. In Annual Conference and General Meeting, 1992, Zoological Society of Bangladesh. Institute of Food and Radiation Biology, Atomic Energy Research Establishment, Savar, Dhaka, 14 January, 1993.

Mandal, H. P. 1995. Studies on helminth parasite of lizardfish, Saurida tumbil (Bloch,1795). An M.Sc. thesis submitted to the Department of Fisheries Biology and Limnology, BAU, Mymensingh. 75 pp.

Mohanta, S. K. 1995. Investigation of monogenean gill parasites of certain Puntius spp. of Mymensingh. Unpublished [MS Thesis], Department of Aquaculture, BAU, Mymensingh. pp. 59-63.

Mohanta, S. K. and Chandra, K. J. 2000. Monogenean infestation in Thai Silver barb (Barbodes gonionotus Bleeker) and their adaptations in Bangladesh waters. Bangladesh J Fish Res. 3, 147-155.

Mohanta, S. K., Chandra, K. J. and Hossain, M. M. 2000. Dactylogyrid monogeneans from two Puntius species of Mymensingh, Bangladesh. Riv Di Parassitol 61, 209-216.

Moravec, F. 1998. Nematode of freshwater fishes of the Neotropical Region. Academia Praha 4, 64.

Muniruzzan, M., Jahura, U. A. and Chowdhury, M. B. R. 2002. Occurrence of ich in carp nursery ponds and its chemotherapy. Banglades Veterinarian 19, 48-53.

Rahman, A. K. A. 1967. Fish lice and mortality. Agril News(Krishikatha) 27, 22-24.

Rahman, A. K. A. 1968. A note on Argulus species which caused mortality in carps in the experimental cistern of the Freshwater research Station, Chandpur, East Pakistan. Pakistan J Sci Indust Res 11, 115-118.

Rahman, A. K. A. and Ali, M. Y. 1968. The incidence of namatode, Procamallanus heteropneustus in the stomach of Heteropneutes fossilis. Pakistan J Sci Indust Res 11, 112-113.

Rashid, M. M., Aminul Haque, A. K. M. and Chandra, K. J. 1983. Records of some metazoan parasites of Clarias batrachus (Linnaeus) from Mymensingh. Bangladesh JFish 6, 37 - 42

Rashid, M. M., Aminul Haque, A. K. M. and Chowdhury, M. B. R. 1985. Population dynamics of caryophyllid cestodes parasitizing Clarias batrachus. Bangladesh J Agril Sci 12, 169-174.

Rashid, M. M.., Aminul Haque, A. K. M. and Chandra, K. J. 1984. Effect of season, sex and size of Clarias batrachus on the population of Orientocreadium batrachoides in Mymensingh, Bangladesh. Bangladesh $\mathrm{J}$ Fish 7, 21-25.

Saha, P. K., Chandra. K. J. and Ghosh, P. K. 2003. Monogenean parasites of certain small indigenous fish species of Bangladesh. Riv. Di Parassit. 64, 203-215.

Sanaullah, M. 1984. On the occurrence of black spot and fish leech in Bangladesh waters. Bangladesh J Fish 7, 81-83.

Sanaullah, M. 1993. Research priorities from the DoF perspective. In "Research Priorities in Bangladesh for Fish Health, Disease Prevention and Pathology" (Tollervey, Ed.), A one-day ODA/BAU workshop held at the Faculty of Fisheries, BAU, Mymensingh, 17 May, 1993. pp. 17-19.

Sanaullah, M. and Ahmed, A. T. A. 1980. Gill myxoboliasis of major carps in Bangladesh. J Fish Dis 3, 349-354.

Uddin, M, Dewan, M. L., Hossain, M. I. and Huq, M. M. 1980. Occurrence of Diphyllobothrium latus larvae (plerocercoid) in loitya (Harpodon nehereus) fish. Bangladesh Vet J14, 33-35. 